On the other hand, some other manufacturers use receiver bandwidths considerably less than these values which, while it confers benefits in terms of target detectability (lower KTB noise), may also lead to failure to observe frequency agile RACONS due to the RACON reply frequency error causing the reply to be outside the radar receiver passband for longer displayed range scales.

Reflections from the surface of a smooth sea can also cause the fading of RACON responses. This effect, which can also adversely affect radar echoes and is known as Lloyd's Mirror, is dependent upon the range of the RACON from the radar and also upon the heights of the antennas of the radar and RACON above the sea surface.

The visibility of RACON responses at closer range can be affected by the improper setting of the radar anti-clutter-sea (STC) controls, since, if the sea clutter suppression threshold is set too high, it may also suppress RACON or other beacon replies.

4. FUTURE DEVELOPMENTS. A new development in marine navigation radar technology which has implications for operation with standard RACONs, is the Signaal scout radar, now being tested by the Dutch and Canadian navies. scour is a cw radar having a peak transmitted power of I W compared with the $25 \mathrm{~kW}$ of a standard marine navigation radar. The low transmitted power, together with the $\mathrm{FM}-\mathrm{CW}$ mode of operation, means that standard marine navigation RACONS, which require a pulsed signal, will not be triggered.

Low peak transmitted powers are environmentally attractive and it seems likely that scout points the way for the future evolution of commercial marine navigation radar, provided that the problem of incompatibility with RACONS and Search and Rescue Transponders (SART), can be overcome.

KEY WORDS

I. Radar, 2. Radar Beacons.

\title{
A Step by Step Method of Computing Points Along the Arc of a Great Circle
}

\author{
Roy Williams
}

I. INTRODUCTION. On any surface with suitable continuity properties, the shortest distance between two points on the surface is along the arc of a geodesic curve. A geodesic curve on such a surface is defined as a curve on the surface along whose length, at any point, the normal to the curve is also the normal to the surface at that point. On the surface of a sphere, the geodesic arcs are the great circles and, except when the two points are antipodean, the arc of the great circle joining them is unique.

In navigation the standard procedure for computing great circle course and distance, and any intermediate points along the path, employs the methods of spherical trigonometry. These methods, which usually involve the haversine formula and the spherical cosine formula, are well tried and tested and give accurate results but they are not sufficiently general and cannot be applied to surfaces other than the sphere. They can be used to provide first approximations to the path of the geodesic on the surface of the 
Terrestial Spheroid and in the correction methods of Andoyer ${ }^{1}$ and Lambert ${ }^{2}$ but, if we were to consider a surface other than the Earth, such as Jupiter, where the flattening of the surface is much more distinct then, although the methods of spherical trigonometry might still provide first approximations to the properties of the geodesic arcs, some other method must provide the final answer.

It is the purpose here, using the methods of differential geometry, to derive an equation in spherical coordinates for a great circle on the surface of a sphere from which, at any point $\mathrm{P}$ along the path of the great circle where the longitude is $\theta$, we can find the latitude, $\phi$, of the point P. $\phi$ and $\theta$ are defined in the ranges

$$
-\frac{1}{2} \pi<\phi<\frac{1}{2} \pi \text { (North positive) : } 0<\theta<2 \pi \text { (East positive) }
$$

The method finds Clairaut's Equation which defines a geodesic curve on any surface which is also a surface of revolution.

Using Clairaut's Equation and the Direct Cubic Spline Approximation to Integrals ${ }^{3}$ we will also describe a method of computing the course and distance along the path of a great circle which can be generalized to other surfaces of revolution and which, even in the case of the sphere, might be considered a more straightforward alternative to the methods of spherical trigonometry.

2. CLAIRAUT'SEQUATION. A sphere is an example of a special set of surfaces known as surfaces of revolution. A surface of revolution is formed by revolving a curve through $360^{\circ}$ around a fixed axis. A sphere, for instance, is formed by revolving a circle about a diameter. On any surface of revolution which satisfies the required continuity conditions, the special set of curves formed by the intersection of the surface with planes through the axis of revolution are known as meridians. Meridians are geodesic curves.

Consider, then, a geodesic curve on a surface of revolution which passes through a set of points $\left[P_{i}\right]$ and which cuts the meridian through a particular point $P_{i}$ at an angle $\gamma_{i}$ (see Fig. I). If $r_{i}$ is the shortest distance from the point $P_{i}$ to the axis of revolution, along

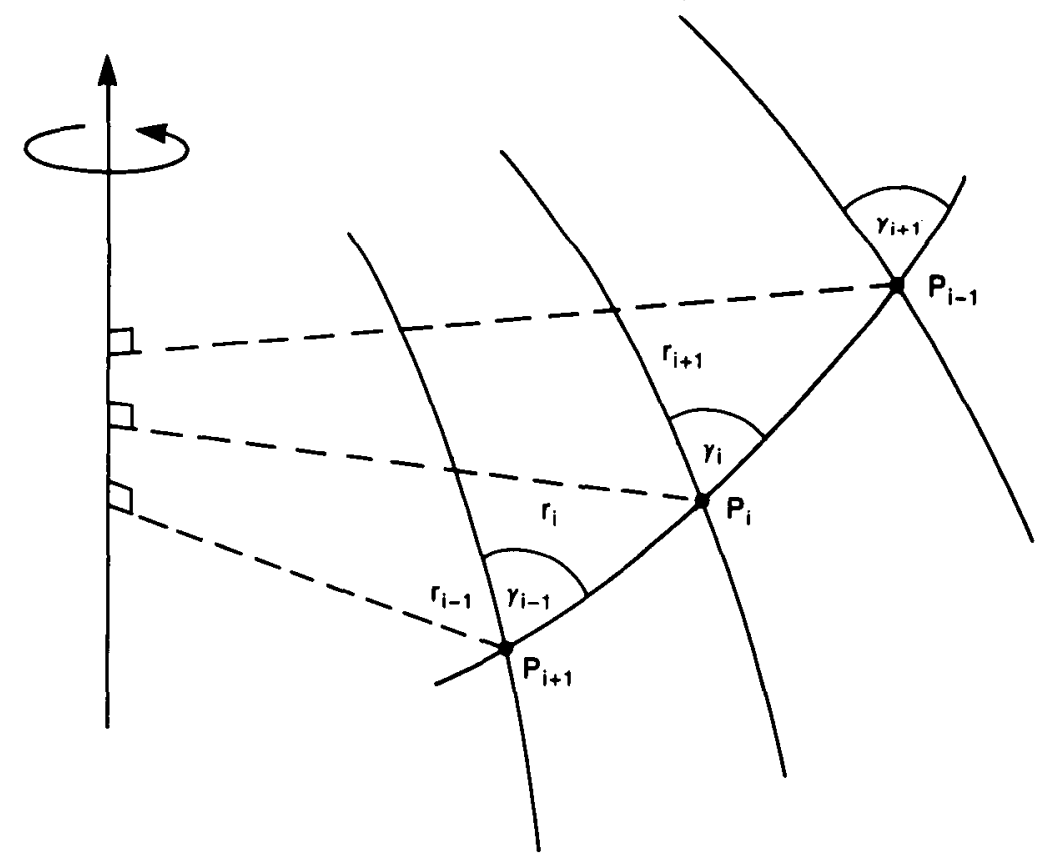

Fig. 1 . 
a line perpendicular to the axis of revolution, then for all points $\mathrm{P}_{i}$ on the geodesic, Clairaut's Equation is

$$
r_{i} \sin \gamma_{i}=\text { constant }
$$

This equation is shown in the book by Lyusternik. ${ }^{4}$

If we consider a small element, $\delta s$, of the great circle arc on the surface of a sphere of radius $a$ at a point $\mathrm{P}$ along its path then, by the methods of differential geometry, as $\delta s$ approaches zero, we can define a limiting triangle PQR (Fig. 2), right-angled at $\mathrm{R}$, in which $\mathrm{PQ}=d s ; \mathrm{PR}=a \cos \phi d \theta ; \mathrm{QR}=a d \phi ; \mathrm{PQR}=\gamma$

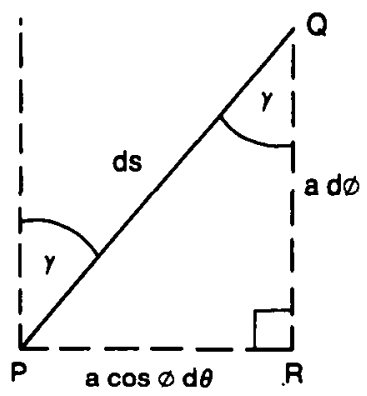

Fig. 2.

where $\gamma$ is the angle at which the great circle cuts the meridian at $P$. The distance from the point $P$ along a line perpendicular to the axis of revolution is $a \cos \phi$ and, from triangle $P Q R$, we see that

$$
\sin \gamma=\frac{a \cos \phi d \theta}{d s}
$$

so that, when the surface of revolution is a sphere, Clairaut's Equation may be expressed as a differential equation in the form

$$
a^{2} \cos ^{2} \phi\left(\frac{d \theta}{d s}\right)=\text { constant }
$$

Now, at the vertex of a great circle, where the latitude is $\phi_{\mathrm{v}}$, say, the angle $\gamma=90^{\circ}$, $\sin \gamma=1$ so that $r=a \cos \phi_{\mathrm{v}}$ and this is the value of the constant. Hence, using this in equation (3), Clairaut's Equation becomes

$$
a \cos ^{2} \phi\left(\frac{d \theta}{d s}\right)=\cos \phi_{\mathrm{v}}
$$

Using the relationship $\quad d s^{2}=a^{2} d \phi^{2}+a^{2} \cos ^{2} \phi d \theta^{2}$

and the substitution $y=a \tan \phi$ we can transform equation (4) to

$$
\mathrm{d} \theta=\frac{d y}{\sqrt{ }\left(y_{\mathrm{v}}^{2}-y^{2}\right)}
$$

Hence, when the great circle crosses the Equator in longitude $\theta_{e}$, its solution is

$$
y=y_{\mathrm{v}} \sin \left(\theta-\theta_{e}\right)
$$


Replacing $y$ with $a \tan \phi$, we then find

$$
\tan \phi=\tan \phi_{\mathrm{v}} \sin \left(\theta-\theta_{e}\right)
$$

This is the equation of a great circle on the surface of a sphere. We can find the values of $\phi_{v}$ and $\theta_{e}$ for a particular great circle. If the great circle passes through the points $P_{0}$, where the latitude is $\phi_{0}$ and the longitude is $\theta_{0}$, and the point $\mathrm{P}_{n}$, where the latitude is $\phi_{n}$ and the longitude is $\theta_{n}$, then we find, using equation (6)

$$
\tan \theta_{e}=\frac{\tan \phi_{0} \sin \theta_{n}-\tan \phi_{n} \sin \theta_{0}}{\tan \phi_{0} \cos \theta_{n}-\tan \phi_{n} \cos \theta_{0}}
$$

and

$$
\begin{aligned}
& \tan \phi_{\mathrm{v}}=\tan \phi_{0} \operatorname{cosec}\left(\theta_{0}-\theta_{e}\right) \\
& \tan \phi_{\mathrm{v}}=\tan \phi_{n} \operatorname{cosec}\left(\theta_{n}-\theta_{e}\right)
\end{aligned}
$$

using $(8 a)$ or $(8 b)$ in whichever of $\phi_{0}$ or $\phi_{n}$ is non-zero.

The problem of determining from equation ( 7$)$ the value of $\theta_{e}$ which suits the situation is not as straightforward using automatic computation as it is by inspection. For a start, when $\theta_{0}>\theta_{n}$, it is better to rename $\mathrm{P}_{0}$ and $\mathrm{P}_{n}$ temporarily in the computer program so that $\theta_{0}<\theta_{n}$ and distance along the path of the great circle is always computed travelling eastwards. The program must then allow for the fact that, when $\mathrm{P}_{0}$ and $\mathrm{P}_{n}$ lie in opposite hemispheres, the value of $\theta_{e}$ obtained from equation (7) must be such that $\theta_{0}<\theta_{e}<\theta_{n}$. If, however, both $\mathrm{P}_{0}$ and $\mathrm{P}_{n}$ lie in the same hemisphere then the value of $\theta_{e}$ must be the largest value such that $\theta_{e}<\theta_{0}$.

When the computations are complete the computer program will restore all the original information and present the final results in the required order.

3. COMPUTING COURSE AND DISTANCE. At any point along the path of a great circle where the latitude is $\phi$ we can find the course as angle, $\gamma$, from Equation ( $\mathrm{r}$ ) expressed in the form

$$
\sin \gamma=\cos \phi_{\mathrm{v}} \sec \phi
$$

where $\phi_{\mathrm{v}}$ is the latitude of the vertex of the great circle. The distance, $s_{i}$, between the points $\mathrm{P}_{0}$ (latitude $\phi_{0}$, longitude $\theta_{0}$ ) and a point $\mathrm{P}_{i}$ (latitude $\phi_{i}$, longitude $\theta_{i}$ ) along the path of the great circle can be found by separating the variables in Equation (4) and integrating.

We have

$$
s_{i}=\int_{\theta_{0}}^{\theta_{i}} \frac{a \cos ^{2} \phi}{\cos \phi_{v}} d \theta
$$

Now $\phi$ is a function of $\theta$ and they are related by Equation (6) but, even though the integration is with respect to $\theta$, we do not need to express the integrand in terms of $\theta$ since we will use a numerical method to perform the integration and will only need ordinate values at a finite number of points. The method of integration we will use is the direct cubic spline approximation to integrals. ${ }^{3}$

4. A NUMERICAL EXAMPLE. An an example of the application of the above analysis to the computation of intermediate points along the path of a great circle on the surface of a sphere, the course angle at the points and the distance between them, let us consider the arc of the great circle which starts at the Equator in longitude $0^{\circ}$ and reaches its vertex in latitude $45^{\circ}$, longitude $90^{\circ}$.

At a point $\mathrm{P}$ on this particular great circle arc, the latitude, $\phi$, and the longitude, $\theta$, are related by

$$
\tan \phi=\sin \theta
$$

This is a consequence of equation (6). 
Let us choose a set of intermediate points $\left[\mathrm{P}_{i}\right]$ along the path of this great circle arc where the longitudes $\left[\theta_{i}\right]$ of these points are evenly spaced at $5^{\circ}$ intervals. The latitudes $\left[\phi_{i}\right]$ are given by

$$
\phi_{i}=\tan ^{-1}\left(\sin \theta_{i}\right)
$$

At the point $\mathrm{P}_{i}$ the integrand, $f_{i}$, in equation ( 10 ) is, in this case, given by

$$
f_{i}=a \sqrt{ } 2 \cos ^{2} \phi_{i}
$$

and the course angle, $\gamma_{i}$, is, from equation (9), given by

$$
\gamma_{i}=\sin ^{-1}\left(\frac{1}{2} \sqrt{ } 2 \sec \phi_{i}\right)
$$

If $S_{i}$ is the computed value of the distance $\mathrm{P}_{0} \mathrm{P}_{i}$ along the arc of the great circle and the $\left[M_{i}\right]$ are the 'Moments' of the cubic spline which are the computed values of the derivative of the integrand

$$
M_{i} \approx d f_{i} / d \theta
$$

then the step-by-step computational scheme for finding the latitudes of the points along the path, the course angles and the distances is given by

$$
S_{0}=0: f_{0}=a \sqrt{ } 2: M_{0}=0: h=\pi / 36: a=3437.7468
$$

For $i=1$ to 18 :

$$
\begin{aligned}
\theta_{i} & =i h \\
\phi_{i} & =\tan ^{-1}\left(\sin \theta_{i}\right) \\
\gamma_{i} & =\sin ^{-1}\left(\frac{1}{2} \sqrt{ } 2 \sec \phi_{i}\right) \\
f_{i} & =a \sqrt{ } 2 \cos ^{2} \phi_{i} \\
M_{i} & =\frac{2\left(f_{i}-f_{i-1}\right)}{h}-M_{i-1} \\
S_{i} & =S_{i-1}+\frac{h}{2}\left(f_{i}+f_{i-1}\right)-\frac{h^{2}}{12}\left(M_{i}-M_{i-1}\right)
\end{aligned}
$$

The results from the computation are shown in Table 1 . The distances are given in units of $\mathrm{I}$ min of arc on the Equator.

5. CONCLUSION. The results shown in Table I are given correct to two decimal places. The course angle is, in fact, correct to all the available accuracy on the computer in use (1 0 digits) and the final distance is correct to five decimal places before rounding.

It is the belief of this author (with much experience at sea in calculating great circle data) that there is less computation in the method presented here than with the methods using spherical trigonometry and that, although a particularly straightforward example was chosen to demonstrate the method, the computational scheme of Section 4 is suitable for both manual and automatic computation. As pointed out in the introduction, the method here has the advantage that it can be generalized to other surfaces which are also surfaces of revolution and, indeed, this was done (though not explicitly) in the paper by Williams and Phythian, ${ }^{5}$ where the shortest distance step by step along a geodesic arc on the surface of a spheroid was computed. When used in an automatic mode this method can also be adapted to update once an observed position has been found, and the great circle track to the destination can be re-evaluated very quickly using the same intermediate longitudes. When interfaced with a position fixing system the updating procedure can, of course, be almost continuous giving the course along the tangent to the great circle and the distance to the destination. 
TABLE I

\begin{tabular}{|c|c|c|c|c|c|}
\hline & $i$ & $\theta_{i}$ & $\phi_{i}$ & Course & Distance \\
\hline & I & $5^{\circ}$ & $4^{\circ} 5^{8} \cdot 9^{\prime}$ & $045^{\circ} 22^{\circ}$ & 423.20 \\
\hline & 2 & $10^{\circ}$ & $9^{\circ} 5 I^{\prime} I^{\prime}$ & $045^{\circ} 86^{\circ}$ & $840^{\circ} \cdot 2$ \\
\hline & 3 & $15^{\circ}$ & $14^{\circ} 30.6^{\prime}$ & $046.92^{\circ}$ & $1245^{.22}$ \\
\hline & 4 & $20^{\circ}$ & $18^{\circ} 52.9^{\prime}$ & $048 \cdot 36^{\circ}$ & $1634^{\circ} 19$ \\
\hline & 5 & $25^{\circ}$ & $22^{\circ} 54^{\circ} 6^{\prime}$ & $050 \cdot 14^{\circ}$ & $2004 \cdot 20$ \\
\hline & 6 & $30^{\circ}$ & $26^{\circ} 33^{\circ} 9^{\prime}$ & $052.24^{\circ}$ & 2353.90 \\
\hline & 7 & $35^{\circ}$ & $29^{\circ} 50^{\circ} 3^{\prime}$ & $0.54 \cdot 60^{\circ}$ & 2683.15 \\
\hline & 8 & $40^{\circ}$ & $3^{\circ} 43^{\circ} 9^{\prime}$ & $057^{\circ} 20^{\circ}$ & 2992.76 \\
\hline & 9 & $45^{\circ}$ & $35^{\circ} 15^{\circ} 9^{\prime}$ & $060.00^{\circ}$ & $3284: 14$ \\
\hline & 10 & $50^{\circ}$ & $37^{\circ} 27^{\prime} 2^{\prime}$ & $062.97^{\circ}$ & 3559.08 \\
\hline & II & $55^{\circ}$ & $39^{\circ} 19^{\prime} 4^{\prime}$ & $066.07^{\circ}$ & 3819.54 \\
\hline & I 2 & $60^{\circ}$ & $40^{\circ} 53^{\prime} 6^{\prime}$ & $069.30^{\circ}$ & 4067.54 \\
\hline & 13 & $65^{\circ}$ & $42^{\circ}$ II' $2^{\prime}$ & $072 \cdot 6 \mathrm{I}^{\circ}$ & 4305.07 \\
\hline & I 4 & $70^{\circ}$ & $43^{\circ} 13^{\prime} 2^{\prime}$ & $076.00^{\circ}$ & 4534.03 \\
\hline & 15 & $75^{\circ}$ & $44^{\circ} 004^{\prime}$ & $079.45^{\circ}$ & 4756.28 \\
\hline & 16 & $80^{\circ}$ & $44^{\circ} 337^{\prime}$ & $082.95^{\circ}$ & 4793.56 \\
\hline & 17 & $85^{\circ}$ & $44^{\circ} 53 \cdot 4^{\prime}$ & $086.47^{\circ}$ & $5187 \cdot 60$ \\
\hline & 18 & $90^{\circ}$ & $45^{\circ} 00 \cdot 0^{\prime}$ & $090.00^{\circ}$ & 5400.00 \\
\hline
\end{tabular}

\section{REFEREN CES}

1 Andoyer, H. (1932). Formule donnat le longeur entre deux pointes de l'ellipsoide donnes par leur coordonnés géographiques. Bulletin Geodésique 34, 77.

${ }^{2}$ Lambert, W. (1942). The distance between two widely separated points on the surface of the Earth. Journal of the Washington Academy of Science 32, 125.

${ }^{3}$ Phythian, J. \& Williams, R. (1985). Direct cubic spline approximation to an integral. Bulletin of the Institute of Mathematics and its Applications, 21 (July/August).

4 Lyusternik, L. A. (1964). Shortest Paths. Pergamon Press.

3 Williams, R. \& Phythian, J. E. (1989). Navigating along geodesic paths on the surface of a spheroid. This Journal, 42 (129).

KEY WORDS

I. Geodesy.

\section{Fast-Ferry Navigation}

Dag Pike

Recent accidents to fast ferries, notably that to Sea Cat in Norway, have concentrated attention on fast-ferry operations, and the navigation of this new generation of fast craft. The Sea Cat accident was a navigation error which resulted in the vessel running on to rocks with the deaths of two passengers, and brought into sharp focus the risks which can be inherent in fast-ferry operations. It is suggested that prior to this accident there have probably been many other navigation errors which have had the potential for serious consequences. This has prompted a look at the problems and limitations of current high-speed navigation practices and possible solutions to reduce the chance of accidents amongst the expanding fleet of high-speed ferries. This paper also proposes a method by which standards of equipment and performance could be established in order to provide a better basis for the evaluation of safety margins. 